\title{
Analysis of a stochastic SIR model with fractional Brownian motion
}

\author{
Tomás Caraballo $^{1}$, Sami Keraani ${ }^{2}$ \\ ${ }^{1}$ Departamento de Ecuaciones Diferenciales y Análisis Numérico, \\ Facultad de Matemáticas, Universidad de Sevilla \\ c/ Tarfia s/n, 41012-Sevilla, Spain \\ caraball@us.es \\ ${ }^{2}$ Faculty of Sciences, University of Sfax - Tunisia \\ samikeraani@hotmail.com
}

\begin{abstract}
In this article, a stochastic version of a SIR nonautonomous model previously introduced in [11] is considered. The noise considered is a fractional Brownian motion which satisfies the property of long range memory, which roughly implies that the decay of stochastic dependence with respect to the past is only subexponentially slow, what makes this kind of noise a realistic choice for problems with long memory in the applied sciences. The stochastic model containing a standard Brownian motion has been studied in [5].

In this paper we analyse the existence and uniqueness of solutions to our stochastic model as well as their positiveness.
\end{abstract}

Keywords: SIR model, epidemiology, fractional Brownian motion.

\section{Introduction}

The analysis of several biological models containing nonautonomous or stochastic perturbations has been intensively investigated over the last years (see, for instance, [6], [7], [8] and the references therein). In the nondeterministic case, most used strategies consist in considering stochastic 
or random perturbations. These approaches require different techniques and also provide different kind of results. Also, several types of noise can be considered in the models and each of them allows to highlight some properties which are inherent in the real phenomena.

In this paper we will consider a stochastic perturbation of the following deterministic SIR model:

$$
\left\{\begin{array}{l}
\dot{S}(t, \omega)=q-a S(t)+b I(t)-\gamma \frac{S(t) I(t)}{N(t)} \\
\dot{I}(t, \omega)=-(a+b+c) I(t)+\gamma \frac{S(t) I(t)}{N(t)} \\
\dot{R}(t, \omega)=c I(t)-a R(t)
\end{array}\right.
$$

where $q, a, b, c, \gamma$ are nonnegative constants (see [4] for more details about the model and the previously mentioned constants). In [11] the authors analyse a nonautonomous version of (1.1) (see also [10]), in fact they consider the case in which the per capita infection rate varies in time. This can be modeled by introducing a forcing term which can be either time dependent (see [11]) or random.

In [5], the authors consider a case in which the forcing term is nondeterministic and can be modeled in two different ways: first, one of the parameters is a random coefficient and they study the problem in the framework of Random Dynamical Systems, and second, they consider a stochastic perturbation of system (1.1) by using a standard Wiener process or Brownian motion.

However, in the current paper we will consider a different stochastic perturbation. Instead of a standard Brownian motion we will use a fractional Brownian motion (fBm) $B^{H}$ with Hurst parameters $H \in(1 / 2,1)$. In probability theory, an $\mathrm{fBm}$ is a centered Gaussian process with a special covariance function determined by the Hurst parameter $H \in(0,1)$. For $H=1 / 2, B^{1 / 2}$ is the standard Brownian motion where its generalised temporal derivative is the white noise. For $H \neq 1 / 2, B^{H}$ is not a semi-martingale and, as a consequence, classical techniques of Stochastic Analysis are not applicable. Moreover, it is not a Markov process. In particular, an fBm with a Hurst parameter $H \in(1 / 2,1)$ possesses a property of long range memory, which roughly implies that the decay of stochastic dependence with respect to the past is only subexponentially slow, what makes this kind of noise a realistic choice for problems with long memory in the applied sciences, and this is why this kind of noise is being used now very often.

In this paper, we will assume that one of the parameters in (1.1) is affected by some perturbation driven by an $\mathrm{fBm} B^{H}$. For simplicity and clarity, we only assume that one parameters is affected by the noise, although all of them could experience the same effect. In this way, we will change parameter $a$ by $a-\sigma \dot{B}^{H}$ (see below for more details about $\mathrm{fBm}$ ), consequently, we will consider

$$
\left\{\begin{array}{l}
\dot{S}(t, \omega)=q-a S(t)+b I(t)-\gamma \frac{S(t) I(t)}{N(t)}+\sigma S(t) \dot{B}_{t}^{H}, \\
\dot{I}(t, \omega)=-(a+b+c) I(t)+\gamma \frac{S(t) I(t)}{N(t)}+\sigma I(t) \dot{B}_{t}^{H}, \\
\dot{R}(t, \omega)=c I(t)-a R(t)+\sigma R(t) \dot{B}_{t}^{H}
\end{array}\right.
$$

First we will analyse the existence and uniqueness of solutions to the this model. Next, we 
will prove that the biological relevant solutions are always in the positive octant, in other words, that any solution starting in the positive octant will remain there forever. Due to the nature of the stochastic perturbation, the axial equilibrium $(q / a, 0,0)$ is not longer an equilibrium for the stochastic system, so it does not make sense to analyse its stability properties.

The structure of this paper is as follows. In Section 2 we recall some preliminary results about fractional Brownian motions and other auxiliary results which will be necessary for our study. Section 3 is devoted to the analysis of the existence and uniqueness of solutions to our stochastic SIR model, while the positiveness of those solutions is proved in Section 4. Some conclusions and open research directions will be included in Section 5 .

\section{Preliminaries}

In this section we recall some notations and necessary concepts for our objectives (see [3]). Let us start by considering two given values $T_{1}<T_{2}$. Let $C^{\beta}\left(\left[T_{1}, T_{2}\right], \mathbb{R}^{3}\right)$ be the Banach space of Hölder continuous functions with exponent $0<\beta<1$ with values in $\mathbb{R}^{3}$, and norm

$$
\|u\|_{\beta, \rho, T_{1}, T_{2}}=\|u\|_{\infty, \rho, T_{1}, T_{2}}+\|u\|_{\beta, \rho, T_{1}, T_{2}}
$$

where $\rho \geq 0$ and

$$
\begin{aligned}
\|u\|_{\infty, \rho, T_{1}, T_{2}} & =\sup _{s \in\left[T_{1}, T_{2}\right]} e^{-\rho\left(s-T_{1}\right)}\|u(s)\| \\
\|u\|_{\beta, \rho, T_{1}, T_{2}} & =\sup _{T_{1} \leq s<t \leq T_{2}} e^{-\rho\left(t-T_{1}\right)} \frac{\|u(t)-u(s)\|}{(t-s)^{\beta}} .
\end{aligned}
$$

For $\rho>0$ and $\rho=0$ the corresponding norms are equivalent. We will suppress the index $\rho$ in these notations if $\rho=0$, and we will suppress $T_{1}, T_{2}$ when $T_{1}=0$ and $T_{2}=1$.

In order to define integrals with Hölder-continuous integrators, we next define Weyl fractional derivatives of functions on separable Hilbert spaces (see [14]).

Definition 2.1. Let $V_{1}$ and $V_{2}$ be separable Hilbert spaces and let $0<\alpha<1$. The Weyl fractional derivatives of general measurable functions $Z:[s, t] \rightarrow V_{1}$ and $\omega:[s, t] \rightarrow V_{2}$, of order $\alpha$ and $1-\alpha$ respectively, are defined for $s<r<t$ by

$$
\begin{aligned}
D_{s+}^{\alpha} Z[r] & =\frac{1}{\Gamma(1-\alpha)}\left(\frac{Z(r)}{(r-s)^{\alpha}}+\alpha \int_{s}^{r} \frac{Z(r)-Z(q)}{(r-q)^{1+\alpha}} d q\right) \in V_{1}, \\
D_{t-}^{1-\alpha} \omega_{t-}[r] & =\frac{(-1)^{\alpha}}{\Gamma(\alpha)}\left(\frac{\omega(r)-\omega(t-)}{(t-r)^{1-\alpha}}+(1-\alpha) \int_{r}^{t} \frac{\omega(r)-\omega(q)}{(q-r)^{2-\alpha}} d q\right) \in V_{2},
\end{aligned}
$$

where

$$
\omega_{t-}(r)=\omega(r)-\omega(t-),
$$

and $\omega(t-)$ is the left side limit of $\omega$ at $t$. 
The next result shows that Weyl fractional derivatives are well-posed for Hölder-continuous functions with suitable Hölder exponents. The proof follows easily and therefore we omit it.

Lemma 2.1. Suppose that $Z \in C^{\beta}\left(\left[T_{1}, T_{2}\right] ; V_{1}\right)$ and $\omega \in C^{\beta^{\prime}}\left(\left[T_{1}, T_{2}\right] ; V_{2}\right), T_{1} \leq s<t \leq T_{2}$ and that $0<\alpha<\beta$ and $\alpha+\beta^{\prime}>1$. Then $D_{s+}^{\alpha} Z$ and $D_{t-}^{1-\alpha} \omega_{t-}$ are well defined.

Let us assume for a while that $V_{1}=V_{2}=\mathbb{R}$. Following Zähle [15] we can define the fractional integral given by

$$
\int_{s}^{t} Z d \omega=(-1)^{\alpha} \int_{s}^{t} D_{s+}^{\alpha} Z[r] D_{t-}^{1-\alpha} \omega_{t-}[r] d r .
$$

We collect some properties of these integrals, for the proof see [9] and [15].

Lemma 2.2. Let $Z, Z_{1}, Z_{2} \in C^{\beta}\left(\left[T_{1}, T_{2}\right] ; \mathbb{R}\right), \omega, \omega_{1}, \omega_{2} \in C^{\beta^{\prime}}\left(\left[T_{1}, T_{2}\right] ; \mathbb{R}\right)$ such that $\beta^{\prime}>1-\beta$. Then there exists a constant $C_{\beta, \beta^{\prime}}$ such that for $T_{1} \leq s<t \leq T_{2}$

$$
\left|\int_{s}^{t} Z d \omega\right| \leq C_{\beta, \beta^{\prime}}\left(1+(t-s)^{\beta}\right)(t-s)^{\beta^{\prime}}\|Z\|_{\beta, T_{1}, T_{2}}\|\omega\|_{\beta^{\prime}, T_{1}, T_{2}} .
$$

In addition,

$$
\begin{aligned}
& \int_{s}^{t}\left(Z_{1}+Z_{2}\right) d \omega=\int_{s}^{t} Z_{1} d \omega+\int_{s}^{t} Z_{2} d \omega \\
& \int_{s}^{t} Z d\left(\omega_{1}+\omega_{2}\right)=\int_{s}^{t} Z d \omega_{1}+\int_{s}^{t} Z d \omega_{2} .
\end{aligned}
$$

The integral is additive: for $\tau \in[s, t]$

$$
\int_{s}^{t} Z d \omega=\int_{s}^{\tau} Z d \omega+\int_{\tau}^{t} Z d \omega
$$

Let $\left(\omega_{n}\right)_{n \in \mathbb{N}}$ be a sequence converging in $C^{\beta^{\prime}}\left(\left[T_{1}, T_{2}\right] ; \mathbb{R}\right)$ to $\omega$. Then we have

$$
\lim _{n \rightarrow \infty}\left\|\int_{T_{1}}^{\cdot} Z d \omega_{n}-\int_{T_{1}}^{\cdot} Z d \omega\right\|_{\beta, T_{1}, T_{2}}=0
$$

We now extend the definition of a fractional integral in $\mathbb{R}$ to a fractional integral in the separable Hilbert space $\mathbb{R}^{3}$, following the construction carried out recently in [9] in a general separable Hilbert-space. To do that, consider the separable Hilbert space $L_{2}\left(\mathbb{R}^{3}\right)$ of HilbertSchmidt operators from $\mathbb{R}^{3}$ into $\mathbb{R}^{3}$. Let $Z \in C^{\beta}\left(\left[T_{1}, T_{2}\right] ; L_{2}\left(\mathbb{R}^{3}\right)\right)$ and $\omega \in C^{\beta^{\prime}}\left(\left[T_{1}, T_{2}\right] ; \mathbb{R}^{3}\right)$ with $\beta^{\prime}>1-\beta$. We define the $\mathbb{R}^{3}$-valued integral for $T_{1} \leq s<t \leq T_{2}$ as

$$
\int_{s}^{t} Z d \omega:=(-1)^{\alpha} \sum_{j=1}^{3}\left(\sum_{i=1}^{3} \int_{s}^{t} D_{s+}^{\alpha}\left\langle\mathbf{e}_{j}, Z(\cdot) \mathbf{e}_{i}\right\rangle[r] D_{t-}^{1-\alpha}\left\langle\mathbf{e}_{i}, \omega(\cdot)\right\rangle_{t-}[r] d r\right) \mathbf{e}_{j}
$$


with $\left\{\mathbf{e}_{1}, \mathbf{e}_{2}, \mathbf{e}_{3}\right\}$ is the basis of the space $\mathbb{R}^{3}$.

for $1-\beta^{\prime}<\alpha<\beta$, whose norm fulfills

$$
\left\|\int_{s}^{t} Z d \omega\right\| \leq \int_{s}^{t}\left\|D_{s+}^{\alpha} Z[r]\right\|_{L_{2}\left(\ell^{2}\right)}\left\|D_{t-}^{1-\alpha} \omega_{t-}[r]\right\| d r .
$$

Note that in (2.1) the integrals under the sums are one-dimensional fractional integrals. In particular, in [9] the following result was proved:

Theorem 2.3. Suppose that $Z \in C^{\beta}\left(\left[T_{1}, T_{2}\right] ; L_{2}\left(\mathbb{R}^{3}\right)\right)$ and $\omega \in C^{\beta^{\prime}}\left(\left[T_{1}, T_{2}\right] ; \mathbb{R}^{3}\right)$ where $\beta+\beta^{\prime}>1$. Then there exists $\alpha \in(0,1)$ such that $1-\beta^{\prime}<\alpha<\beta$ and the integral (2.1) is well defined. Moreover, all properties of Lemma 2.2 hold if we replace the $\mathbb{R}$-norm by the $\mathbb{R}^{3}$-norm.

We now consider estimates of the integral with respect to the Hölder norms depending on $\rho$.

Lemma 2.4. Under the assumptions of Theorem 2.3, for $\beta^{\prime}>\beta$ there exists a constant $c$ depending on $T_{1}, T_{2}, \beta, \beta^{\prime}$ such that for $T_{1} \leq s<t \leq T_{2}$

$$
e^{-\rho t}\left\|\int_{s}^{t} Z d \omega\right\| \leq c k(\rho)\|Z\|_{\beta, \rho, s, t}\|\omega\|_{\beta^{\prime}, s, t}(t-s)^{\beta},
$$

such that $\lim _{\rho \rightarrow \infty} k(\rho)=0$. Moreover, for any $\tau \in \mathbb{R}$

$$
\int_{s}^{t} Z(r) d \omega(r)=\int_{s-\tau}^{t-\tau} Z(r+\tau) d \theta_{\tau} \omega(r)
$$

Proof. We only sketch the proof, for more details see [9].

First of all, it is not difficult to see that

$$
\left\|D_{t-}^{1-\alpha} \omega_{t-}[r]\right\| \leq c\|\omega\|_{\beta^{\prime}, s, t}(t-r)^{\alpha+\beta^{\prime}-1} .
$$

Furthermore, since $Z \in C^{\beta}\left(\left[T_{1}, T_{2}\right] ; L_{2}\left(\mathbb{R}^{3}\right)\right)$,

$$
\begin{aligned}
e^{-\rho t}\left\|D_{s+}^{\alpha} Z[r]\right\|_{L_{2}\left(\ell^{2}\right)} & \leq c e^{-\rho(t-r)}\left(e^{-\rho r} \frac{\|Z(r)\|_{L_{2}\left(\ell^{2}\right)}}{(r-s)^{\alpha}}+\int_{s}^{r} e^{-\rho r} \frac{\|Z(r)-Z(q)\|_{L_{2}\left(\ell^{2}\right)}}{(r-q)^{1+\alpha}} d q\right) \\
& \leq c e^{-\rho(t-r)}\|Z\|_{\beta, \rho, s, t}\left((r-s)^{-\alpha}+(r-s)^{\beta-\alpha}\right) \\
& \leq c e^{-\rho(t-r)}\left(1+(r-s)^{\beta}\right)\|Z\|_{\beta, \rho, s, t}(r-s)^{-\alpha} .
\end{aligned}
$$

Therefore,

$$
\begin{aligned}
& e^{-\rho t}\left\|\int_{s}^{t} Z d \omega\right\| \leq c\|\omega\|_{\beta^{\prime}, s, t}\|Z\|_{\beta, \rho, s, t} \int_{s}^{t} e^{-\rho(t-r)}(t-r)^{\alpha+\beta^{\prime}-1}(r-s)^{-\alpha} d r \\
& \quad \leq c\|\omega\|_{\beta^{\prime}, s, t}\|Z\|_{\beta, \rho, s, t}(t-s)^{\beta} \int_{s}^{t} e^{-\rho(t-r)}(t-r)^{\alpha+\beta^{\prime}-\beta-1}(r-s)^{-\alpha} d r \\
& \quad \leq c k(\rho)\|\omega\|_{\beta^{\prime}, s, t}\|Z\|_{\beta, \rho, s, t}(t-s)^{\beta},
\end{aligned}
$$


where

$$
k(\rho)=\sup _{s<t \in[0, T]} \int_{s}^{t} e^{-\rho(t-r)}(t-r)^{\alpha+\beta^{\prime}-\beta-1}(r-s)^{-\alpha} d r
$$

is such that $\lim _{\rho \rightarrow \infty} k(\rho)=0$. The previous property can be stated in general as follows: given $T>0$, if $a, b>-1$ are such that $a+b+1>0$, then

$$
k(\rho):=\sup _{s<t \in[0, T]} \int_{s}^{t} e^{-\rho(t-r)}(r-s)^{a}(t-r)^{b} d r,
$$

is such that $\lim _{\rho \rightarrow \infty} k(\rho)=0$, see [9].

From now on $k(\rho)$ will denote a function with the above behavior no matter the exact values of the corresponding parameters $a, b>-1$ provided that $a+b+1>0$. Moreover, note that the above constraints imply that $\beta^{\prime}>1 / 2$.

As a particular case of Hölder-continuous integrator we are going to consider a fractional Brownian motion (fBm) with values in $\mathbb{R}^{3}$ with Hurst-parameter $H>1 / 2$. Consider a probability space $(\Omega, \mathcal{F}, \mathbb{P})$. Let $\left(B_{i}^{H}\right)_{i \in \mathbb{Z}}$ be an iid-sequence of fBm with the same Hurst-parameter $H>1 / 2$ over this probability space, that is, each $B_{i}^{H}$ is a centered Gauß-process on $\mathbb{R}$ with covariance

$$
R(s, t)=\frac{1}{2}\left(|s|^{2 H}+|t|^{2 H}-|t-s|^{2 H}\right) \quad \text { for } s, t \in \mathbb{R} .
$$

Let $Q$ be a linear operator on $\mathbb{R}^{3}$ such that $Q \mathbf{e}_{i}=\sigma_{i}^{2} \mathbf{e}_{i}, \sigma=\left(\sigma_{i}\right)_{i=1,2,3}$. Hence $Q$ is a non-negative and symmetric operator of trace class. A continuous $\mathbb{R}^{3}$-valued $\mathrm{fBm} B^{H}$ with covariance operator $Q$ and Hurst parameter $H$ is defined by

$$
B^{H}(t)=\sum_{i=1}^{3}\left(\sigma_{i} B_{i}^{H}(t)\right) \mathbf{e}_{i}
$$

having covariance

$$
R_{Q}(s, t)=\frac{1}{2} Q\left(|s|^{2 H}+|t|^{2 H}-|t-s|^{2 H}\right) \quad \text { for } s, t \in \mathbb{R} .
$$

In fact, since the process $B^{H}$ is a Gauß-process

$$
\begin{aligned}
\mathbb{E}\left\|B^{H}(t)-B^{H}(s)\right\|^{2}= & \sum_{i \in \mathbb{Z}} \sigma_{i}^{2} \mathbb{E}\left(B_{i}^{H}(t)-B_{i}^{H}(s)\right)^{2}=\sum_{i \in \mathbb{Z}} \sigma_{i}^{2}|t-s|^{2 H}=\|\sigma\|^{2}|t-s|^{2 H}, \\
& \mathbb{E}\left\|B^{H}(t)-B^{H}(s)\right\|^{2 n} \leq c_{n}|t-s|^{2 H n} .
\end{aligned}
$$

Therefore, applying Kunita [12] Theorem 1.4.1, $B^{H}(t)$ has a continuous version and also a Hölder-continuous version with exponent less than $H$, see Bauer [2] Chapter 39. Note that $B^{H}(0)=0$ almost surely. 
Let $C_{0}\left(\mathbb{R} ; \mathbb{R}^{3}\right)$ be the space of continuous functions on $\mathbb{R}$ with values in $\mathbb{R}^{3}$ which are zero at zero, equipped with the compact open topology. Then let $\left(C_{0}\left(\mathbb{R} ; \mathbb{R}^{3}\right), \mathcal{B}\left(C_{0}\left(\mathbb{R} ; \mathbb{R}^{3}\right)\right), \mathbb{P}_{H}\right)$ be the canonical space for $\mathrm{fBm}$, i.e. $B^{H}(\omega)(t)=\omega(t)$, where $\mathbb{P}_{H}$ denotes the measure of the fBm with Hurst-parameter $H$. On $C_{0}\left(\mathbb{R} ; \mathbb{R}^{3}\right)$ we can introduce the Wiener shift $\theta$ given by the measurable flow

$$
\theta:\left(\mathbb{R} \times C_{0}\left(\mathbb{R}, \mathbb{R}^{3}\right), \mathcal{B}(\mathbb{R}) \otimes \mathcal{B}\left(C_{0}\left(\mathbb{R}, \mathbb{R}^{3}\right)\right)\right) \rightarrow\left(C_{0}\left(\mathbb{R}, \mathbb{R}^{3}\right), \mathcal{B}\left(C_{0}\left(\mathbb{R}, \mathbb{R}^{3}\right)\right)\right)
$$

such that

$$
\theta(t, \omega)(\cdot)=: \theta_{t} \omega(\cdot)=\omega(\cdot+t)-\omega(t)
$$

By Mishura [13] we have that $\theta_{t}$ leaves $\mathbb{P}_{H}$ invariant. In addition $t \rightarrow \theta_{t} \omega$ is continuous.

Furthermore, thanks to Bauer [2] Chapter 39, we can also conclude that the set $C_{0}^{\beta^{\prime}}\left(\mathbb{R} ; \mathbb{R}^{3}\right)$ of continuous functions which have a finite $\beta^{\prime}-$ Hölder-seminorm on any compact interval and which are zero at zero has $\mathbb{P}_{H^{-}}$-measure one for $\beta^{\prime}<H$, is $\theta$-invariant.

\section{$3 \quad$ Existence and uniqueness}

In order to prove the result on the existence and uniqueness of solutions to our model, we will first observe that we can rewrite it in an abstract form as the following SIR epidemic model

$$
d u(t)=[A u(t)+F(u(t))] d t+\sigma_{0}(t) u(t) d B^{H}(t)
$$

where $A$ is an operator (matrix) which generates a uniformly continuous semigroup $S_{A}(t):=e^{-t A}$, and $A$ is given by

$$
A=\left(\begin{array}{ccc}
-a & b & 0 \\
0 & -(a+b+c) & 0 \\
0 & c & -a
\end{array}\right)
$$

and the other terms are given by

$$
F(u)=\left(\begin{array}{c}
q-\frac{\gamma u_{1} u_{2}}{u_{1}+u_{2}+u_{3}} \\
\frac{\gamma u_{1} u_{2}}{u_{1}+u_{2}+u_{3}} \\
0
\end{array}\right), \quad \sigma_{0}(t)=\sigma
$$

We emphasize that the relevant domain for our analysis is the positive octant $\mathbb{R}_{+}^{3}=\left\{\left(x_{1}, x_{2}, x_{3}\right) \in\right.$ $\left.\mathbb{R}^{3}: x_{i} \geq 0, i=1,2,3\right\}$, and in this region the function $F$ is globally Lipschitz. Consequently, we will proceed with a general Lipschitz function $F$ with Lipschitz constant $L_{F}$, instead of our 
particular case, and in this way we will be covering other interesting examples in our more abstract formulation. Also we will consider a more general time variable coefficient $\sigma_{0}(\cdot)$ (but bounded, say, by a constant $\sigma$ ) instead of the constant $\sigma$ in our problem.

Lemma 3.1. The uniformly continuous semigroup $S_{A}(\cdot)$ is exponentially stable, that is,

$$
\left\|S_{A}\right\| \leq e^{-a t}, \quad \text { for } t \geq 0 \text {. }
$$

In addition, for $0 \leq s<t$

$$
\begin{aligned}
\left\|S_{A}(t)-S_{A}(s)\right\| & \leq\|A\|(t-s) e^{-a s}, \\
\left\|S_{A}(t-s)-i d\right\| & \leq\|A\|(t-s) .
\end{aligned}
$$

The proof of the first property is a direct consequence of the energy inequality, while the two last estimates follow easily by the mean value theorem.

It is also straightforward to see that the matrix $A$ is diagonalizable and there exist two matrices $P$ and $D$ such that

$$
A=P D P^{-1}
$$

with

$$
D=\left(\begin{array}{ccc}
-a & 0 & 0 \\
0 & -a & 0 \\
0 & 0 & -(a+b+c)
\end{array}\right)
$$

and

$$
P=\left(\begin{array}{ccc}
1 & 0 & \frac{b}{b+c} \\
0 & 0 & 1 \\
0 & 1 & \frac{c}{b+c}
\end{array}\right)
$$

Then, $S_{A}(t)=e^{t A}=P e^{t D} P^{-1}$ and it is easy to check again that

$$
\left\|S_{A}\right\| \leq\left\|P e^{t D} P^{-1}\right\| \leq e^{-a t}
$$

As straightforward consequences, we also obtain that for $0<s<t$,

$$
\left\|S_{A}(t-.)\right\|_{\beta, 0, T}=\sup _{0 \leq r_{1}<r_{2} \leq T} \frac{\left\|S_{A}\left(t-r_{2}\right)-S_{A}\left(t-r_{1}\right)\right\|}{\left(r_{2}-r_{1}\right)^{\beta}} \leq\|A\| t^{1-\beta}
$$

and

$$
\begin{aligned}
\| S_{A}(t-.) & -S_{A}(s-.) \|_{\beta, 0, T} \\
& =\sup _{0 \leq r_{1}<r_{2} \leq T} \frac{\left\|\left(S_{A}(t-s)-i d\right)\left(S_{A}\left(s-r_{2}\right)-S_{A}\left(s-r_{1}\right)\right)\right\|}{\left(r_{2}-r_{1}\right)^{\beta}} \\
& \leq\|A\|^{2}(t-s) s^{1-\beta} .
\end{aligned}
$$


Theorem 3.2. Equation (3.1) has a unique solution $\left.u \in C^{\beta}([0, T]) ; \mathbb{R}^{3}\right)$.

Proof. We will show that the operator

$$
X_{u_{0}, \omega}(u)[t]=S_{A}(t) u_{0}+\int_{0}^{t} S_{A}(t-r) F(u(r)) d r+\int_{0}^{t} S_{A}(t-r) \sigma_{0}(r) u(r) d \omega(r),
$$

where $t \in[0, T]$, possesses a unique fixed point in $C^{\beta}\left([0, T] ; \mathbb{R}^{3}\right)$ by applying the Banach fixed point theorem. To this end, first of all we show that there exists a closed centered ball with respect to the norm $\|\cdot\|_{\beta, \rho, 0, T}$ which is mapped by $X_{u_{0}, \omega}$ into itself. For the first term, in virtue of (3) we obtain

$$
\left\|S_{A}(.) u_{0}\right\|_{\beta, \rho, 0, T} \leq\left(1+\|A\| T^{1-\beta}\right)\left\|u_{0}\right\| .
$$

For the Lebesgue integral of $X_{u_{0}, \omega}$ we obtain

$$
\begin{aligned}
\left\|\int_{0} S_{A}(.-r) F(u(r)) d r\right\|_{\beta, \rho, 0, T} & \leq \sup _{t \in[0, T]} e^{-\rho t}\left\|\int_{0}^{t} S_{A}(t-r) F(u(r)) d r\right\| \\
& +\sup _{0 \leq s<t \leq T} e^{-\rho t} \frac{\left\|\int_{s}^{t} S_{A}(t-r) F(u(r)) d r\right\|}{(t-s)^{\beta}} \\
& +\sup _{0 \leq s<t \leq T} e^{-\rho t} \frac{\left\|\int_{0}^{s}\left(S_{A}(t-r)-S_{A}(t-r)\right) F(u(r)) d r\right\|}{(t-s)^{\beta}}
\end{aligned}
$$

For the first term, we have

$$
\begin{aligned}
\sup _{t \in[0, T]} e^{-\rho t}\left\|\int_{0}^{t} S_{A}(t-r) F(u(r)) d r\right\| & \leq \sup _{t \in[0, T]} \int_{0}^{t} e^{-\rho(t-r)} d r\|F(u(.))\|_{\infty, \rho, 0, T} \\
& \leq \frac{1}{\rho}\|F(u(.))\|_{\infty, \rho, 0, T} .
\end{aligned}
$$

For the second,

$$
\begin{aligned}
\sup _{0 \leq s<t \leq T} e^{-\rho t} \frac{\left\|\int_{s}^{t} S_{A}(t-r) F(u(r)) d r\right\|}{(t-s)^{\beta}} & \leq \sup _{0 \leq s<t \leq T} \frac{\int_{s}^{t} e^{-\rho(t-r)} d r}{(t-s)^{\beta}}\|F(u(.))\|_{\infty, \rho, 0, T} \\
& \leq\left(\frac{1}{\rho^{1-\beta}} \frac{1-e^{-\rho(t-s)}}{\rho^{\beta}(t-s)^{\beta}}\right)\|F(u(.))\|_{\infty, \rho, 0, T} \\
& \leq\left(\frac{1}{\rho^{1-\beta}} \sup _{x>0} \frac{1-e^{-x}}{x^{\beta}}\right)\|F(u(.))\|_{\infty, \rho, 0, T} \\
& \leq\left(c_{\beta} \frac{1}{\rho^{1-\beta}}\right)\|F(u(.))\|_{\infty, \rho, 0, T} .
\end{aligned}
$$


And for the third,

$$
\begin{aligned}
& \sup _{0 \leq s<t \leq T} e^{-\rho t} \frac{\left\|\int_{0}^{s}\left(S_{A}(t-r)-S_{A}(t-r)\right) F(u(r)) d r\right\|}{(t-s)^{\beta}} \\
= & \sup _{0 \leq s<t \leq T} e^{-\rho t} \frac{\left\|\int_{0}^{s}\left(\left(S_{A}(t-s)-I d\right) S_{A}(t-r)\right) F(u(r)) d r\right\|}{(t-s)^{\beta}} \\
\leq & \sup _{0 \leq s<t \leq T} e^{-\rho t}\left\|\int_{0}^{s}\left(S_{A}(t-r)\right) F(u(r)) d r\right\|\|A\|(t-s)^{(1-\beta)} \\
\leq & \left.\sup _{0 \leq s<t \leq T}\left\|\int_{0}^{s} e^{-\rho(t-r)} d r\right\|\|F(u(r))\|\|A\|(t-s)^{1-\beta}\right) \\
\leq & \frac{1}{\rho}\|F(u(r))\|\|A\| T^{1-\beta} .
\end{aligned}
$$

Then,

$$
\begin{aligned}
\left\|\int_{0} S_{A}(.-r) F(u(r)) d r\right\|_{\beta, \rho, 0, T} & \leq\left(\frac{1}{\rho}+c_{\beta} \frac{1}{\rho^{1-\beta}}+\frac{1}{\rho} T^{1-\beta}\|A\|\right)\|F(u(.))\|_{\infty, \rho, 0, T} \\
& \leq K(\rho)\|F(u(.))\|_{\infty, \rho, 0, T}
\end{aligned}
$$

but

$$
\begin{aligned}
\|F(u(.))\|_{\infty, \rho, 0, T} & \leq \sup _{0 \leq t \leq T} e^{-\rho t}\left\|F\left(u_{0}\right)\right\|+e^{-\rho t}\left\|F(u(t))-F\left(u_{0}\right)\right\| \\
& \leq\left\|F\left(u_{0}\right)\right\|+L_{F} T^{\beta}\|u\|_{\beta, \rho, 0, T}
\end{aligned}
$$

hence

$$
\left\|\int_{0}^{\cdot} S_{A}(.-r) F(u(r)) d r\right\|_{\beta, \rho, 0, T} \leq \hat{K}(\rho)\left(1+\|u\|_{\beta, \rho, 0, T}\right)
$$

On the other hand,

$$
\left\|\int_{0} S_{A}(.-r) \sigma_{0}(r) u(r) d r\right\|_{\beta, \rho, 0, T} \leq \sigma k(\rho)\left\|B^{H}\right\|_{\beta^{\prime}, 0, T} \leq\left(1+\|u(r)\|_{\beta, \rho, 0, T}\right),
$$

In conclusion, we have obtained

$$
\left\|X_{u_{0}, \omega}(u)\right\|_{\beta, \rho, 0, T} \leq\left(1+\|A\| T^{1-\beta}\right)\left\|u_{0}\right\|+K(\rho)\left(1+\|\omega\|_{\beta^{\prime}, 0, T}\right)\left(1+\|u\|_{\beta, \rho, 0, T}\right)
$$

where $\lim _{\rho \rightarrow \infty} K(\rho)=0$. Note that $K(\rho)$ may also depend on the parameters related to $F$, $\|A\|$ and $T$. Taking a sufficiently large $\rho$ such that $K(\rho)\left(1+\|\omega\|_{\beta^{\prime}, 0, T}\right) \leq \frac{1}{2}$, the ball

$$
B=B\left(0, R\left(u_{0}, \rho\right)\right)=u \in C^{\beta}\left([0, T] ; \mathbb{R}^{3}\right):\|u\|_{\beta, \rho, 0, T} \leq R
$$

with 


$$
R=R\left(u_{0}, \rho\right)=2\left(1+\|A\| T^{1-\beta}\right)\left\|u_{0}\right\|+1,
$$

is mapped into itself since

$$
\left\|X_{u_{0}, \omega}(u)\right\|_{\beta, \rho, 0, T} \leq\left(1+\|A\| T^{1-\beta}\right)\left\|u_{0}\right\|+\frac{1}{2}(1+R)=R .
$$

We now derive the contraction condition for the operator $X_{u_{0}, \omega}(u)$ with respect to the norm $\|.\|_{\beta, \rho, 0, T}$ where the $\bar{\rho}$ may differ from the $\rho$ considered above. However, since all these norms are equivalent for different $\rho \geq 0$, the set $B$ remains a complete space with respect to any $\|\cdot\|_{\beta, \rho, 0, T}$.

Similar to above, for the Lebesgue integral we obtain the estimate

$$
\left\|F\left(u_{1}(.)\right)-F\left(u_{2}(.)\right)\right\|_{\beta, \bar{\rho}, 0, T} \leq k(\bar{\rho}) L_{F}\left\|u_{1}-u_{2}\right\|_{\beta, \bar{\rho}, 0, T} .
$$

Regarding the stochastic integral, we have

$$
\left\|\sigma_{0}(.) u_{1}(.)-\sigma_{0}(.) u_{2}(.)\right\|_{\beta, \bar{\rho}, 0, T} \leq \sigma\left\|u_{1}(.)-u_{2}(.)\right\|_{\beta, \bar{\rho}, 0, T} .
$$

Then,

$$
\left\|X_{u_{0}, \omega}\left(u_{1}\right)-X_{u_{0}, \omega}\left(u_{2}\right)\right\|_{\beta, \bar{\rho}, 0, T} \leq K(\bar{\rho})\left(1+\|\omega\|_{\beta^{\prime}, 0, T}\right)\left(1+\left\|u_{1}\right\|_{\infty, 0, T}+\left\|u_{2}\right\|_{\infty, 0, T}\right)\left\|u_{1}-u_{2}\right\|_{\infty, \bar{\rho}, 0, T},
$$

where again, $\lim _{\bar{\rho} \rightarrow \infty} K(\bar{\rho})=0$. It suffices then to choose $\bar{\rho}$ sufficiently large so that

$$
\left\|X_{u_{0}, \omega}\left(u_{1}\right)-X_{u_{0}, \omega}\left(u_{2}\right)\right\|_{\beta, \bar{\rho}, 0, T} \leq \frac{1}{2}\left\|u_{1}-u_{2}\right\|_{\beta, \bar{\rho}, 0, T}
$$

which implies the contraction property of the map $X_{u_{0}, \omega}$. Hence, (3.1) has a unique solution $u \in C^{\beta}\left([0, T] ; \mathbb{R}^{3}\right)$.

\section{Positiveness of the solution}

Now we will prove that solutions starting in positive initial data remain positive in the future. This is crucial to justify the well-posedness of the problem. To this end, we will perform a change of variable, namely Theorem 2.1 in [1] (see also [15]).

Let us denote by $Y_{t}^{1}=\int_{0}^{t} \sigma_{0}(s) d B_{s}^{H}$ and $h(t)=G\left(Y_{t}^{1}\right)$ with $G(x)=\exp (-x)$

$$
\begin{aligned}
h(t) & =\exp \left(-\int_{0}^{t} \sigma_{0}(s) d B_{s}^{H}\right) \\
& =\exp (-Y(t)) \\
& =G\left(Y_{t}^{1}\right) \\
& =G(0)+\int_{0}^{t} \frac{d G}{d x}\left(Y_{t}^{1}\right) \sigma_{0}(s) d B_{s}^{H} \\
& =1-\int_{0}^{t} \sigma_{0}(s) h(s) d B_{s}^{H} \\
A & =1-h_{1}(t),
\end{aligned}
$$


and consider $u(\cdot)$, solution of our problem

$$
\begin{aligned}
u(t) & =u_{0}+\int_{0}^{t}(A u(s)+F(u(s))) d s+\int_{0}^{t} \sigma_{0}(s) u(s) d B_{s}^{H} \\
& =u_{0}+u_{1}(t)+u_{2}(t) .
\end{aligned}
$$

Then, by denoting $Z(t)=h(t) u(t)$, we deduce

$$
\begin{aligned}
Z(t) & =h(t) u(t) \\
& =\left(1-h_{1}(t)\right)\left(u_{0}+u_{1}(t)+u_{2}(t)\right) \\
& =u_{0}+u_{1}(t)+u_{2}(t)-u_{0} h_{1}(t)-h_{1}(t) u_{1}(t)-h_{1}(t) u_{2}(t)
\end{aligned}
$$

Now we apply again Theorem 2.1 in [1] by using $G(x, y)=x y$ so that $\frac{\partial G}{\partial y}=x$ and $\frac{\partial G}{\partial x}=y$

$$
\begin{aligned}
h_{1}(t) u_{1}(t) & =G\left(h_{1}(t), u_{1}(t)\right) \\
& =G(0)+\int_{0}^{t} \frac{\partial G}{\partial y}\left(h_{1}(s), u_{1}(s)\right)(A u(s)+F(u(s))) d s \\
& +\int_{0}^{t} \frac{\partial G}{\partial x}\left(h_{1}(s), u_{1}(s)\right) \sigma_{0}(s) h(s) d B_{s}^{H} \\
& =\int_{0}^{t} h_{1}(s)(A u(s)+F(u(s))) d s+\int_{0}^{t} u_{1}(s) \sigma_{0}(s) h(s) d B_{s}^{H} \\
h_{2}(t) u_{2}(t) & =\int_{0}^{t} h_{1}(s) \sigma_{0}(s) u(s) d s+\int_{0}^{t} u_{2}(s) \sigma_{0}(s) h(s) d B_{s}^{H}
\end{aligned}
$$




$$
\begin{aligned}
& Z(t)=u_{0}+u_{1}(t)+u_{2}(t)-u_{0} h_{1}(t)-\int_{0}^{t} h_{1}(s)(A u(s)+F(u(s))) d s \\
& -\int_{0}^{t} u_{1}(s) \sigma_{0}(s) h(s) d B_{s}^{H}-\int_{0}^{t} h_{1}(s) \sigma_{0}(s) u(s) d B_{s}^{H}-\int_{0}^{t} u_{2}(s) \sigma_{0}(s) h(s) d B_{s}^{H} \\
& =u_{0}+u_{1}(t)+u_{2}(t)-u_{0} h_{1}(t)-\int_{0}^{t} h_{1}(s)(A u(s)+F(u(s))) d s \\
& -\int_{0}^{t} h_{1}(s) \sigma_{0}(s) u(s) d B_{s}^{H}-\int_{0}^{t}\left(u_{1}(s)+u_{2}(s)\right) \sigma_{0}(s) h(s) d B_{s}^{H} \\
& =u_{0}+\int_{0}^{t}(A u(s)+F(u(s))) d s+\int_{0}^{t} \sigma_{0}(s) u(s) d B_{s}^{H}-u_{0} h_{1}(t) \\
& -\int_{0}^{t} h_{1}(s)(A u(s)+F(u(s))) d s \\
& -\int_{0}^{t} h_{1}(s) \sigma_{0}(s) u(s) d B_{s}^{H}-\int_{0}^{t}\left(u_{1}(s)+u_{2}(s)\right) \sigma_{0}(s) h(s) d B_{s}^{H} \\
& =u_{0}+\int_{0}^{t}\left(1-h_{1}(s)\right)(A u(s)+F(u(s))) d s+\int_{0}^{t}\left(1-h_{1}(s)\right) \sigma_{0}(s) u(s) d B_{s}^{H} \\
& -u_{0} h_{1}(t)-\int_{0}^{t}\left(u_{1}(s)+u_{2}(s)\right) \sigma_{0}(s) h(s) d B_{s}^{H} \\
& =u_{0}+\int_{0}^{t} h(s)(A u(s)+F(u(s))) d s+\int_{0}^{t} h(s) \sigma_{0}(s) u(s) d B_{s}^{H} \\
& -\int_{0}^{t} u_{0} \sigma_{0}(s) h(s) d B_{s}^{H}-\int_{0}^{t}\left(u_{1}(s)+u_{2}(s)\right) \sigma_{0}(s) h(s) d B_{s}^{H} \\
& =u_{0}+\int_{0}^{t} h(s)(A u(s)+F(u(s))) d s+\int_{0}^{t} h(s) \sigma_{0}(s) u(s) d B_{s}^{H} \\
& -\int_{0}^{t}\left(u_{0}+u_{1}(s)+u_{2}(s)\right) \sigma_{0}(s) h(s) d B_{s}^{H} \\
& =u_{0}+\int_{0}^{t} h(s)(A u(s)+F(u(s))) d s+\int_{0}^{t} h(s) \sigma_{0}(s) u(s) d B_{s}^{H} \\
& -\int_{0}^{t} u(s) \sigma_{0}(s) h(s) d B_{s}^{H} \\
& =u_{0}+\int_{0}^{t}(A u(s)+F(u(s))) h(s) d s \\
& =h(0) u_{0}+\int_{0}^{t}(A u(s) h(s)+h(s) F(u(s))) d s \\
& =Z_{0}+\int_{0}^{t}\left(A Z(s)+h(s) F\left(h^{-1}(s) Z(s)\right)\right) d s,
\end{aligned}
$$


and, therefore, $Z$ is solution to the following differential system:

$$
Z^{\prime}=A Z+h(t) F\left(h^{-1}(t) Z\right)
$$

with the initial value $Z(0)=Z_{0}$. And due to the expression that $A$ and $F$ have, it is straightforward to check that if $Z_{0} \in \mathbb{R}_{+}^{3}$, the corresponding solution starting in $Z_{0}$ at time $t=0$ remains always inside $\mathbb{R}_{+}^{3}$

\section{Conclusions}

We have analyzed a SIR model which is very much used in Epidemiology, but perturbed by some noise modeled by a fractional Brownian motion with Hurst parameter $H>1 / 2$. We have proved the well-posedness of the problem by proving existence and uniqueness of solutions as well as the positiveness of solutions which, due to the nature of the problem, are the only meaningful solutions. The analysis carried out in the paper can be considered as the preliminar one in order to have a more complete study of the model. In a next paper, we plan to analyze the asymptotic behavior of the model, including the existence of random attractor and its internal structure. As far as we know, there are not works on this direction and we plan to analyze it shortly.

Acknowledgement. The research of Tomás Caraballo was partially supported by the projects MTM2015-63723-P (MINECO/ FEDER, EU) and P12-FQM-1492 (Junta de Andalucía).

\section{References}

[1] Jan Bártek, María J. Garrido-Atienza, and Bohdan Maslowski. Stochastic porous media equation driven by fractional Brownian motion. Stoch. Dyn., 13(4):1350010, 33, 2013.

[2] Heinz Bauer. Probability theory, volume 23 of De Gruyter Studies in Mathematics. Walter de Gruyter \& Co., Berlin, 1996. Translated from the fourth (1991) German edition by Robert B. Burckel and revised by the author.

[3] Hakima Bessaih, María J. Garrido-Atienza, Xiaoying Han, and Björn Schmalfuss. Stochastic lattice dynamical systems with fractional noise. SIAM J. Math. Anal., 49(2):1495-1518, 2017.

[4] Fred Brauer and Carlos Castillo-Chavez. Mathematical models for communicable diseases, volume 84 of CBMS-NSF Regional Conference Series in Applied Mathematics. Society for Industrial and Applied Mathematics (SIAM), Philadelphia, PA, 2013.

[5] Tomás Caraballo and Renato Colucci. A comparison between random and stochastic modeling for a SIR model. Commun. Pure Appl. Anal., 16(1):151-162, 2017. 
[6] Tomás Caraballo, Renato Colucci, and Xiaoying Han. Predation with indirect effects in fluctuating environments. Nonlinear Dynam., 84(1):115-126, 2016.

[7] Tomás Caraballo, Xiaoying Han, and Peter Kloeden. Chemostats with time-dependent inputs and wall growth. Appl. Math. Inf. Sci., 9(5):2283-2296, 2015.

[8] Tomás Caraballo, Xiaoying Han, and Peter E. Kloeden. Chemostats with random inputs and wall growth. Math. Methods Appl. Sci., 38(16):3538-3550, 2015.

[9] Yong Chen, Hongjun Gao, María J. Garrido-Atienza, and Björn Schmalfuss. Pathwise solutions of SPDEs driven by Hölder-continuous integrators with exponent larger than 1/2 and random dynamical systems. Discrete Contin. Dyn. Syst., 34(1):79-98, 2014.

[10] P. E. Kloeden and C. Pötzsche. Nonautonomous bifurcation scenarios in SIR models. Math. Methods Appl. Sci., 38(16):3495-3518, 2015.

[11] Peter E. Kloeden and Victor S. Kozyakin. The dynamics of epidemiological systems with nonautonomous and random coefficients,. MESA: Mathematics in Engineering, Science and Aerospace, 2(2), 2011.

[12] Hiroshi Kunita. Stochastic flows and stochastic differential equations, volume 24 of Cambridge Studies in Advanced Mathematics. Cambridge University Press, Cambridge, 1990.

[13] Yuliya S. Mishura. Stochastic calculus for fractional Brownian motion and related processes, volume 1929 of Lecture Notes in Mathematics. Springer-Verlag, Berlin, 2008.

[14] Stefan G. Samko, Anatoly A. Kilbas, and Oleg I. Marichev. Fractional integrals and derivatives. Gordon and Breach Science Publishers, Yverdon, 1993. Theory and applications, Edited and with a foreword by S. M. Nikolskiu, Translated from the 1987 Russian original, Revised by the authors.

[15] M. Zähle. Integration with respect to fractal functions and stochastic calculus. I. Probab. Theory Related Fields, 111(3):333-374, 1998. 